\title{
Study of transient stability for parallel connected inverters in Microgrid system works in stand-alone
}

\author{
F. Andrade ${ }^{1, *}, J^{*}$ Cusido $^{2}$, L. Romeral ${ }^{1}$, J. J. Cárdenas $^{1}$ \\ ${ }^{1}$ Universitat Politécnica de Catalunya, Barcelona, Spain \\ ${ }^{2}$ CTM Centre Tecnologic, Manresa, Spain \\ *Tel: +34937398510, Fax: +34937398016, E-mail:fabio.andrade@mcia.upc.edu
}

\begin{abstract}
Distributed generators systems and Microgrid are becoming more important to increase the renewable energy penetration in the public utility. This paper presents a mathematical model for connected inverters in Microgrid systems with large range variations in operating conditions. No-lineal tools and computer simulations, phase-plane trajectory analysis, method of Lyapunov and bifurcations analysis for evaluate the limits of the small signal models are used, and conclusion suggested utilizing models that can permit to analysis of the system when subjected to a severe transient disturbance such as loss a large load or loss of generation. The study of transient stability for Microgrid systems in stand-alone of the utility grid is useful to improve the design of Microgrid's architecture.
\end{abstract}

Keywords: Microgrid model, Transient Stability, Parallel Inverters, Method of Lyapunov.

\section{Introduction}

Distributed generators systems and Microgrid are becoming more important to increase the renewable energy penetration in the public utility [1-2]. Thus, the use of intelligent power interfaces between the electrical photovoltaic generation source and the grid is required. These interfaces have a final stage consisting of dc/ac inverters, which can be classified in current source inverters (CSI) and voltage-source inverters (VSI). In order to inject current to the grid, CSI are commonly used, while in island or autonomous operation, VSI are needed to maintain the voltage stability [3-5].

A Microgrid can be operated either in grid connected mode or in stand-alone mode. In grid connected mode, most of the system level dynamics are established by the public utility, this is due to the relatively small size of local generators. In stand-alone mode, the system dynamics are established by micro generators themselves [6]. This paper presents an analysis of transient stability of power systems for parallel connected inverter in a Microgrid under autonomous operation.

The power inverters do not present the natural relations between frequency and active output power, neither between output voltage and reactive output power. Therefore, in order to reach stable operation, when the inverters are connected in parallel, these inherent operating conditions must be established by the inverter's control system [7].

Previous analysis of stability of stand-alone systems have been done by means of small signal models $[5,8]$, either assuming an ideal inverter or considering power inverter with a high switching frequency and their closed-loop inner controllers. Simulation and experimental result show that the systems work well when there is a small perturbation, but when the perturbation is bigger, the system could be in unstable operation.

The small signal analysis works with root locus plots Bode diagrams, Nyquist plots, etc., researching the desired dynamic behaviour of the systems. On the contrary, this paper works with no-lineal tools for large signal analysis supported by computer simulations. It is used 
phase-plane trajectory analysis and method of Lyapunov to evaluate the limits of the small signal models. It is suggested the utilization of models that allow to analyze the system when it is subjected to a s evere transient disturbance such as loss of large loads or loss of generation.

\section{A Microgrid Study}

Fig 1 shows the circuit diagram of the Microgrid systems in stand-alone considered in this paper. This system configuration was studied by Duminda, in [8]. It was considered small signal modeling. For simplicity, the system has two inverter connected, but it can be extended for n-number of inverters.

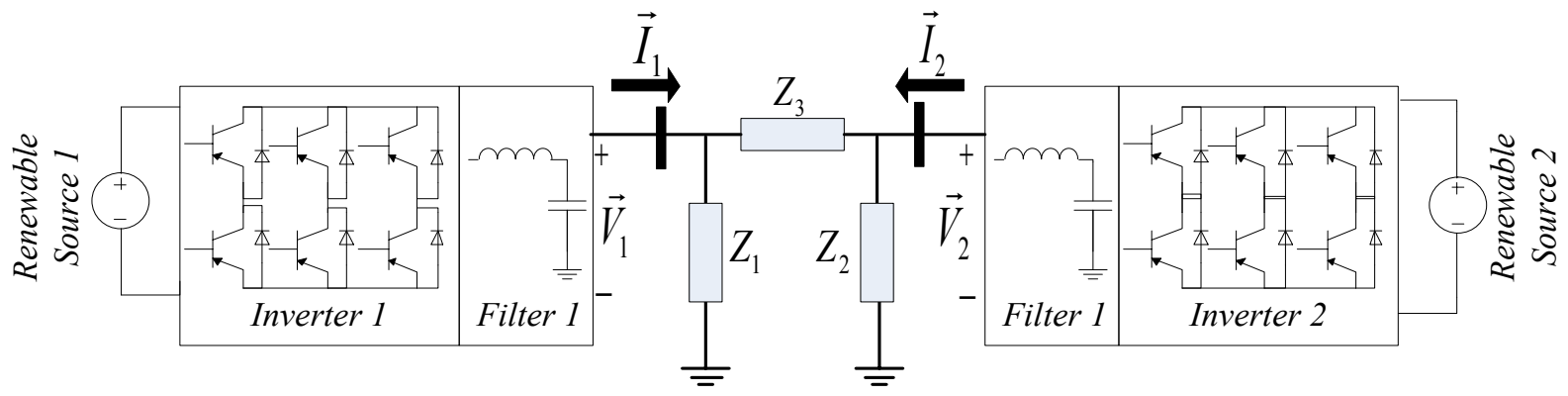

Fig. 1. Parallel connected inverters in Microgrid system works in stand-alone.

The Distributed Generator (DG) considered consists of a power DC renewable source, a three-leg inverter and an output LC filter. By assuming the renewable energy source as an ideal source form, the DC bus dynamics can be neglected. Each DG presents a PWM controller with a current and voltage loop and a PQ controller. The PQ controller must autonomously respond effectively to system changes without communication, only with local variables. Commonly, the PQ controller uses the droop curves $\left(f v_{s} P\right.$ and $\left.V v s Q\right)$ and a filter with a cut-off frequency of approximately a decade smaller than a grid frequency. The task of the PQ controller is to imitate the governor of a synchronous generator. This artificial droop control scheme can be expressed as follows

$\omega=\omega_{0}-k_{p} P \quad V=V_{0}-k_{v} Q$

Where $P$ and $Q$ are filtered with a low pass filter with cut-off frequency $\omega_{\mathrm{f}}$, the equations of these filters in the Laplace domain can be expressed as:

$$
P(s)=\frac{\omega_{f}}{s+\omega_{f}} P_{i}(s) \quad Q(s)=\frac{\omega_{f}}{s+\omega_{f}} Q_{i}(s)
$$

$P_{i}$ and $Q_{i}$ are instantaneous power and are calculated from the measured output voltage and output current as in

$$
P_{i}(s)=V_{d} I_{d}+V_{q} I_{q} \quad Q_{i}(s)=V_{d} I_{q}-V_{q} I_{d}
$$

\subsection{A mathematical model of the each voltage source inverter}

Considering (1), (2) and working in the time domain,

$$
\dot{\omega}=-\omega_{f} \omega-k_{p} \omega_{f}+P_{i}
$$


$\dot{V}=-\omega_{f} V-k_{v} \omega_{f}+Q_{i}$

The inverters are modeled in a common reference frame (d-q), fig. 2. Each voltage vector has an angle $\delta_{\mathrm{i}}$ with respect to the d-axes.

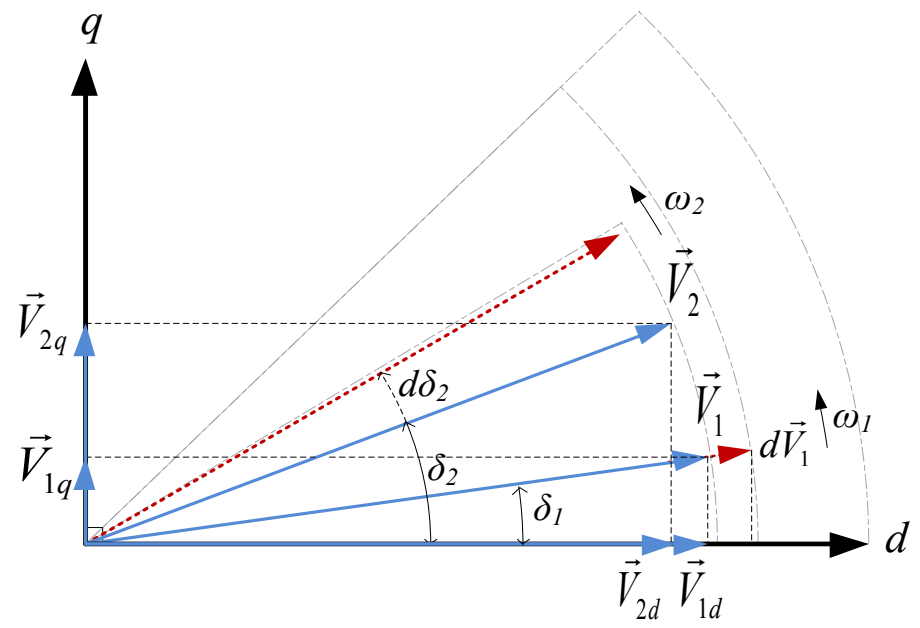

Fig. 2. Voltage vectors in reference d-q frame.

The vector $V$ can be represented as $\vec{V}=V_{d}+j V_{q}$ Where

$$
V_{d}=V \cos (\delta) \quad V_{q}=V \sin (\delta) \quad \delta=\arctan \left(\frac{V_{q}}{V_{d}}\right)
$$

From (4) and fig. $2 \mathrm{it}$ is shown that $\omega_{\mathrm{i}}$ changes in response to the real power flow and modifies the angle $\delta_{i}$. Thus, from (6) it can be expressed

$\omega=\dot{\delta}=\frac{\dot{V}_{q} V_{d}-\dot{V}_{d} V_{q}}{V_{d}{ }^{2}+V_{q}^{2}}$ Considering $|V|=\sqrt{V_{d}^{2}+V_{q}^{2}}$ and the expressions (4), (5) and (6) it can be obtained the state equation (7) which describe the behavior of each inverter.

$$
\left\{\begin{array}{l}
\dot{\omega}=\omega_{f} \omega-k_{p} \omega_{f} P \\
\dot{V}_{d}=-\omega_{f} V_{d}-\omega V_{q}-\frac{k_{p} \omega_{f} V_{d}}{|V|} Q \\
\dot{V}_{q}=\omega V_{d}-\omega_{f} V_{q}--\frac{k_{v} \omega_{f} V_{q}}{|V|} Q
\end{array}\right.
$$

\subsection{Combined model of all the inverters and network model}

In previous section the mathematical model of an individual inverter on a common reference $\mathrm{d}-\mathrm{q}$ frame was discussed. Let us consider a system with two inverters connected to a network, as shown in fig. 1 . The state equation for two inverters is 


$$
\left\{\begin{array}{l}
\dot{\omega}_{1}=\omega_{f 1} \omega_{1}-k_{p 1} \omega_{f 1} P_{1} \\
\dot{V}_{d 1}=-\omega_{f 1} V_{d 1}-\omega_{1} V_{q 1}-\frac{k_{p 1} \omega_{f 1} V_{d 1}}{\left|V_{1}\right|} Q_{1} \\
\dot{V}_{q 1}=\omega_{1} V_{d 1}-\omega_{f 1} V_{q 1}--\frac{k_{v 1} \omega_{f 1} V_{q 1}}{\left|V_{1}\right|} Q_{1} \\
\dot{\omega}_{2}=\omega_{f 2} \omega_{2}-k_{p 2} \omega_{f 2} P_{2} \\
\dot{V}_{d 2}=-\omega_{f 2} V_{d 2}-\omega_{2} V_{q 2}-\frac{k_{p 2} \omega_{f 2} V_{d 2}}{\left|V_{2}\right|} Q_{2} \\
\dot{V}_{q 2}=\omega_{2} V_{d 2}-\omega_{f 2} V_{q 2}--\frac{k_{v 2} \omega_{f 2} V_{q 2}}{\left|V_{2}\right|} Q_{2}
\end{array}\right.
$$

The network in the fig. 1 is described by the nodal admittance matrix equation

$$
\left[\begin{array}{l}
\vec{I}_{1} \\
\vec{I}_{2}
\end{array}\right]=\left[\begin{array}{cc}
Y_{1}+Y_{3} & -Y_{3} \\
-Y_{3} & Y_{2}+Y_{3}
\end{array}\right]\left[\begin{array}{l}
\vec{E}_{1} \\
\vec{E}_{2}
\end{array}\right] \rightarrow\left[\begin{array}{c}
I_{1 d} \\
I_{1 q} \\
I_{2 d} \\
I_{2 q}
\end{array}\right]=\left[\begin{array}{cccc}
G_{11} & -B_{11} & -G_{12} & B_{12} \\
B_{11} & G_{11} & -B_{12} & -G_{12} \\
G_{12} & B_{12} & G_{22} & -B_{22} \\
-B_{12} & -G_{12} & B_{22} & G_{22}
\end{array}\right]\left[\begin{array}{c}
V_{d 1} \\
V_{q 1} \\
V_{d 2} \\
V_{q 2}
\end{array}\right]
$$

Considering the active and reactive power equations (3) and (9),

$$
\left[\begin{array}{l}
P_{1} \\
Q_{1} \\
P_{2} \\
Q_{2}
\end{array}\right]=\left[\begin{array}{cccc}
V_{d 1} & V_{q 1} & 0 & 0 \\
-V_{q 1} & V_{d 1} & 0 & 0 \\
0 & 0 & V_{d 2} & V_{q 2} \\
0 & 0 & -V_{q 2} & V_{d 2}
\end{array}\right]\left[\begin{array}{cccc}
G_{11} & -B_{11} & -G_{12} & B_{12} \\
B_{11} & G_{11} & -B_{12} & -G_{12} \\
G_{12} & B_{12} & G_{22} & -B_{22} \\
-B_{12} & -G_{12} & B_{22} & G_{22}
\end{array}\right]\left[\begin{array}{c}
V_{d 1} \\
V_{q 1} \\
V_{d 2} \\
V_{q 2}
\end{array}\right]
$$

Which the above equation, combined with (8), gives the whole system equations

$$
\begin{aligned}
& \int \dot{X}_{1}=\alpha_{1} X_{1}-\alpha_{2} \sqrt{X_{2}^{2}+X_{3}^{2}}-\alpha_{3} \frac{\left(X_{2} X_{5}+X_{3} X_{6}\right)}{\sqrt{X_{2}^{2}+X_{3}^{2}}}+\alpha_{4} \frac{\left(X_{3} X_{5}-X_{2} X_{6}\right)}{\sqrt{X_{2}^{2}+X_{3}^{2}}} \\
& \dot{X}_{2}=\alpha_{1} X_{2}-X_{1} X_{3}-\alpha_{5} X_{2} \sqrt{X_{2}^{2}+X_{3}^{2}}-\alpha_{6} X_{2} \frac{\left(X_{2} X_{5}+X_{3} X_{6}\right)}{\sqrt{X_{2}^{2}+X_{3}^{2}}}+\alpha_{7} X_{2} \frac{\left(X_{3} X_{5}-X_{2} X_{6}\right)}{\sqrt{X_{2}^{2}+X_{3}^{2}}} \\
& \dot{X}_{3}=\alpha_{1} X_{3}-X_{1} X_{2}-\alpha_{5} X_{3} \sqrt{X_{2}^{2}+X_{3}^{2}}-\alpha_{6} X_{3} \frac{\left(X_{2} X_{5}+X_{3} X_{6}\right)}{\sqrt{X_{2}^{2}+X_{3}^{2}}}+\alpha_{7} X_{3} \frac{\left(X_{3} X_{5}-X_{2} X_{6}\right)}{\sqrt{X_{2}^{2}+X_{3}^{2}}} \\
& \dot{X}_{4}=\beta_{1} X_{4}-\beta_{2} \sqrt{X_{5}^{2}+X_{6}^{2}}-\beta_{3} \frac{\left(X_{2} X_{5}+X_{3} X_{6}\right)}{\sqrt{X_{5}^{2}+X_{6}^{2}}}+\beta_{4} \frac{\left(X_{3} X_{5}-X_{2} X_{6}\right)}{\sqrt{X_{5}^{2}+X_{6}^{2}}} \\
& \dot{X}_{5}=\beta_{1} X_{5}-X_{4} X_{6}-\beta_{5} X_{5} \sqrt{X_{5}^{2}+X_{6}^{2}}-\beta_{6} X_{5} \frac{\left(X_{2} X_{5}+X_{3} X_{6}\right)}{\sqrt{X_{5}^{2}+X_{6}^{2}}}+\beta_{7} X_{5} \frac{\left(X_{3} X_{5}-X_{2} X_{6}\right)}{\sqrt{X_{5}^{2}+X_{6}^{2}}} \\
& \dot{X}_{6}=\beta_{1} X_{6}-X_{4} X_{5}-\beta_{5} X_{6} \sqrt{X_{5}^{2}+X_{6}^{2}}-\beta_{6} X_{6} \frac{\left(X_{2} X_{5}+X_{3} X_{6}\right)}{\sqrt{X_{5}^{2}+X_{6}^{2}}}+\beta_{7} X_{6} \frac{\left(X_{3} X_{5}-X_{2} X_{6}\right)}{\sqrt{X_{5}^{2}+X_{6}^{2}}}
\end{aligned}
$$

Where

$\alpha_{1}=-\omega_{f 1} ; \alpha_{2}=k_{p 1} G_{11} \omega_{f 1} ; \alpha_{3}=k_{p 1} G_{12} \omega_{f 1} ; \alpha_{4}=k_{p 1} B_{12} \omega_{f 1} ; \alpha_{5}=k_{v 1} B_{11} \omega_{f 1} ; \alpha_{6}=k_{v 1} B_{12} \omega_{f 1} ; \alpha_{7}=k_{v 1} G_{12} \omega_{f 1}$ $\beta_{1}=-\omega_{f 2} ; \beta_{2}=k_{p 2} G_{22} \omega_{f 2} ; \beta_{3}=k_{p 2} G_{12} \omega_{f 2} ; \beta_{4}=k_{p 2} B_{12} \omega_{f 2} ; \beta_{5}=k_{v 2} B_{22} \omega_{f 2} ; \beta_{6}=k_{v 2} B_{12} \omega_{f 2} ; \beta_{7}=k_{v 2} G_{12} \omega_{f 2}$ 


\section{Study of Stability for the Microgrid}

In this section it is constructed a Lyapunov function for the Microgrid system and obtained the respective eigenvalues for the A matrix of lineal system.

\subsection{Lyapunov's method}

One of the main impediments for the application of Lyapunov's method to physical systems is the lack of formal procedures to construct the Lyapunov function for the differential equations describing the given physical system [10]. A function $V(X)$ is called a Lyapunov function of the system $f(X)$ where $f(0)=0$ if it fulfills the following properties [11]:

$$
\begin{array}{ll}
\text { i. } & V(0)=0 \\
\text { ii. } & V(X)>0 \text { for all } X \\
\text { iii. } & \frac{d}{d t} V(X) \leq 0 \text { along all trajectories of the system }
\end{array}
$$

Then the point $X=0$ is locally stable.

It is proposed to construct the Lyapunov function by using the set of representative variables for each generator. By following this principle, firstly it is chosen the square frequency variables (X1 and X4) divided into the working frequency to obtain "per unit" values and secondly it is used the dq vol tage of each generator divided by the module of voltage; this method is repeated for every generator of the Microgrid. Finally, every term of the obtained Lyapunov function are multiplied by a different constant. These constants must be chosen to fulfill the iii property.

$$
V(X)=\frac{A X_{1}^{2}}{\omega_{k}}+B \frac{X_{2}^{2}+X_{3}^{2}}{\sqrt{X_{2}^{2}+X_{3}^{2}}}+\frac{C X_{4}^{2}}{\omega_{k}}+D \frac{X_{5}^{2}+X_{6}^{2}}{\sqrt{X_{5}^{2}+X_{6}^{2}}}
$$

The above equation fulfills (i) and (ii) properties as can be seen by inspection. Condition (iii) can be shown as

$$
\frac{\partial V(X)}{\partial X_{1}} \dot{X}_{1}+\frac{\partial V(X)}{\partial X_{2}} \dot{X}_{2}+\frac{\partial V(X)}{\partial X_{3}} \dot{X}_{3}+\frac{\partial V(X)}{\partial X_{4}} \dot{X}_{4}+\frac{\partial V(X)}{\partial X_{5}}+\dot{X}_{5}+\frac{\partial V(X)}{\partial X_{6}} \dot{X}_{6} \leq 0
$$

Hence,

$$
\begin{array}{r}
A X_{1} \dot{X}_{1}+B\left(X_{2}^{2}+X_{3}^{2}\right)^{1 / 2} X_{2} \dot{X}_{2}+C\left(X_{2}^{2}+X_{3}^{2}\right)^{1 / 2}+D X_{3} \dot{X}_{3}+C X_{4} \dot{X}_{4}+ \\
+E\left(X_{5}^{2}+X_{6}^{2}\right)^{1 / 2} X_{5} \dot{X}_{5}+F\left(X_{5}^{2}+X_{6}^{2}\right)^{1 / 2} X_{6} \dot{X}_{6} \leq 0
\end{array}
$$

Then the Lyapunov function (12) and the mathematical model (11) are simulated in MATLAB/Simulink for reaching the best values of every constant and the region where the Lyapunov function is valid and the system is stable. A first study it is used $A=C ; B=E$ and $\mathrm{C}=\mathrm{F}$. 


\subsection{Eigenvalues of lineal system}

Considering the system of the figure 1 and the nonlineal model in (11) and applying Taylor's series around the operating point at this model, it can be obtained the state-space equations of the small-signal model. This is acceptable if variations around the operating point are assumed to be small; therefore, the small signal lineal model is $[\dot{\tilde{X}}]=[A][\tilde{X}]$.

Finally the systems with parameters presented on Table I, the initial active and reactive output powers zero, and initial vectors $\mathrm{V} 1 \mathrm{y} \mathrm{V} 2$ in phase, the A matrix values are

$$
A=\left[\begin{array}{cccccc}
-37.7 & -0.466 & -0.936 & 0 & 0.157 & 0.944 \\
0 & -36.57 & -0.217 & 0 & -0.94 & 0.157 \\
154.5 & 0 & 0 & 0 & 0 & 0 \\
0 & 0.097 & -0.956 & -37.7 & -0.34 & -0.97 \\
0 & -0.954 & 0.097 & -9.86 & -36.5 & -2.42 \\
0 & -0.061 & 0.006 & 154.9 & -2.32 & -0.15
\end{array}\right]
$$

And the respective eigenvalues are $\lambda_{1}=0 ; \lambda_{2}=-10.8 ; \lambda_{3}=-26.9 ; \lambda_{4}=-35.7 \lambda_{5}=-37.7 ; \lambda_{6}=-37.5$; According to [8] only the nonzero eigenvalues are important for the stability studies. The system has five negative real eigenvalues, and then it is a stable point.

Table I. System parameters.

\begin{tabular}{ccc}
\hline Variable & Value & unit \\
\hline Line transmission $\left(Z_{3}\right)$ & $0.5+3 \mathrm{i}$ & $\Omega$ \\
Local load $\left(Z_{1}\right)$ & $13+6 \mathrm{i}$ & $\Omega$ \\
Local load $\left(Z_{2}\right)$ & $25+13 \mathrm{i}$ & $\Omega$ \\
Cut-off freq. of measuring filter $\left(\omega_{f}\right)$ & 37.7 & $\mathrm{rd} / \mathrm{s}$ \\
Frequency droop coefficient $\left(k_{p}\right)$ & 0.0005 & $\mathrm{rd} / \mathrm{s} / \mathrm{W}$ \\
Frequency droop coefficient $\left(k_{v}\right)$ & 0.0005 & $\mathrm{~V} / \mathrm{VAR}$ \\
Nominal frequency & 377 & $\mathrm{rd} / \mathrm{s}$ \\
\hline
\end{tabular}

\section{Simulation Results}

The methodology used is as follows; firstly of all the Microgrid system is implemented be means of the power electronics toolbox of Matlab/SIMULINK, the system parameters for simulation are shown in table I. Secondly, the simulated values of every state variable in the operating point are used to find the A matrix values and its eigenvalues. This eigenvalues are used to determinate the stability of the system. After that, it is utilized the set of equation (11) to verified the model and then the values of the state variables and its derivatives are used to evaluate the Lyapunov function. Finally it is studied the Lyapunov function and it is determined the region of stability of the system.

The fig 3 shows the PQ power simulation by means of the Power electronic toolbox. This was repeated with a different line inductor values (from $8 \mathrm{mH}$ to $0.5 \mathrm{mH}$ with step of $0.5 \mathrm{mH}$ ). For each simulation the values of state variables at the operating point were saved and these were used to study the small signal stability. 

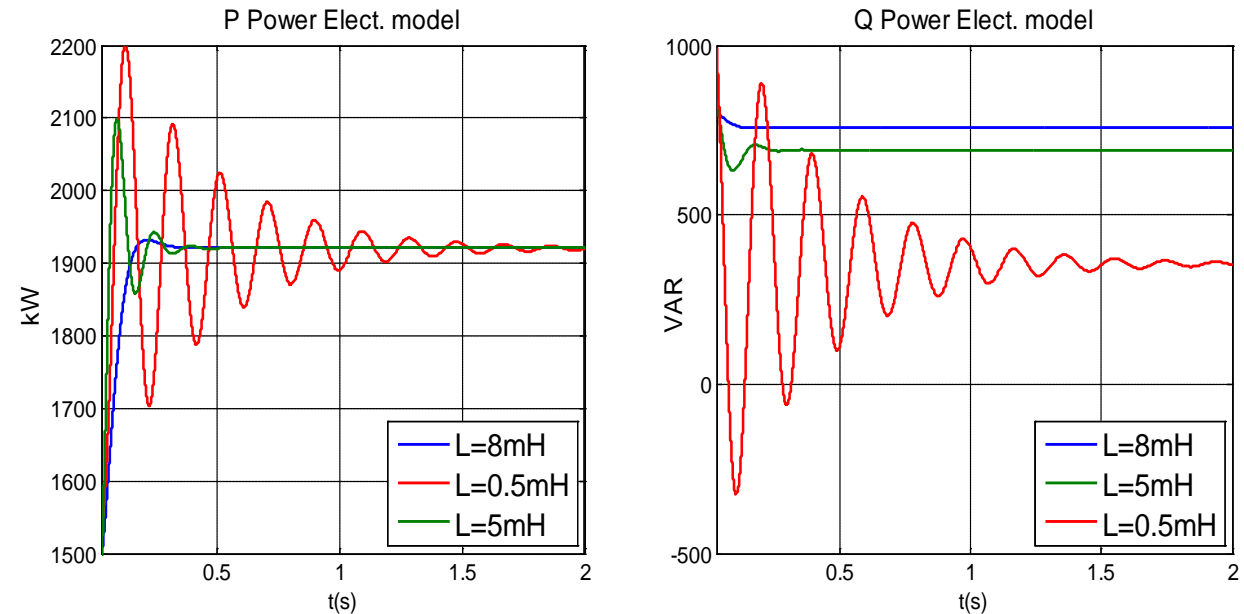

Fig. 3. Simulation results of a DG Microgrid system by means of Power Electronics tools

The fig 4 s hows the PQ power simulation by means of nonlineal model of the Microgrid systems obtained in 2.2. It can be seen the same behavior in both transient and steady state values. As explained previously, the line inductance was varied for the nonlineal model. The values of state variables were used to validate the Lyapunov function.
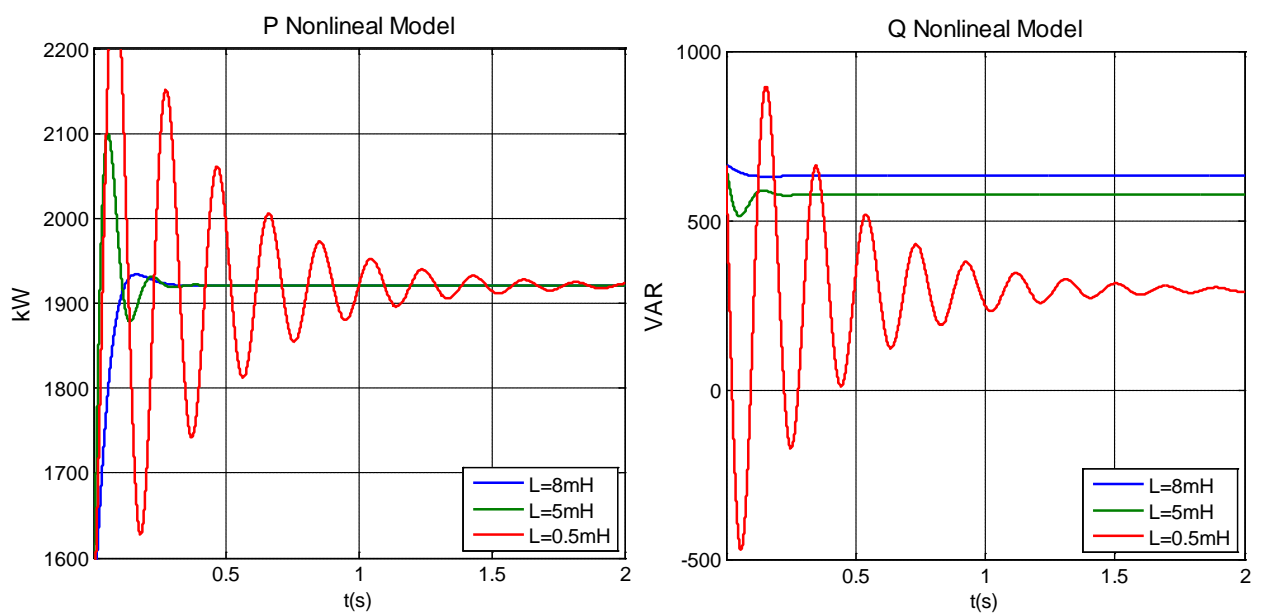

Fig. 4. Simulation results of a DG Microgrid system by means of Nonlineal model.
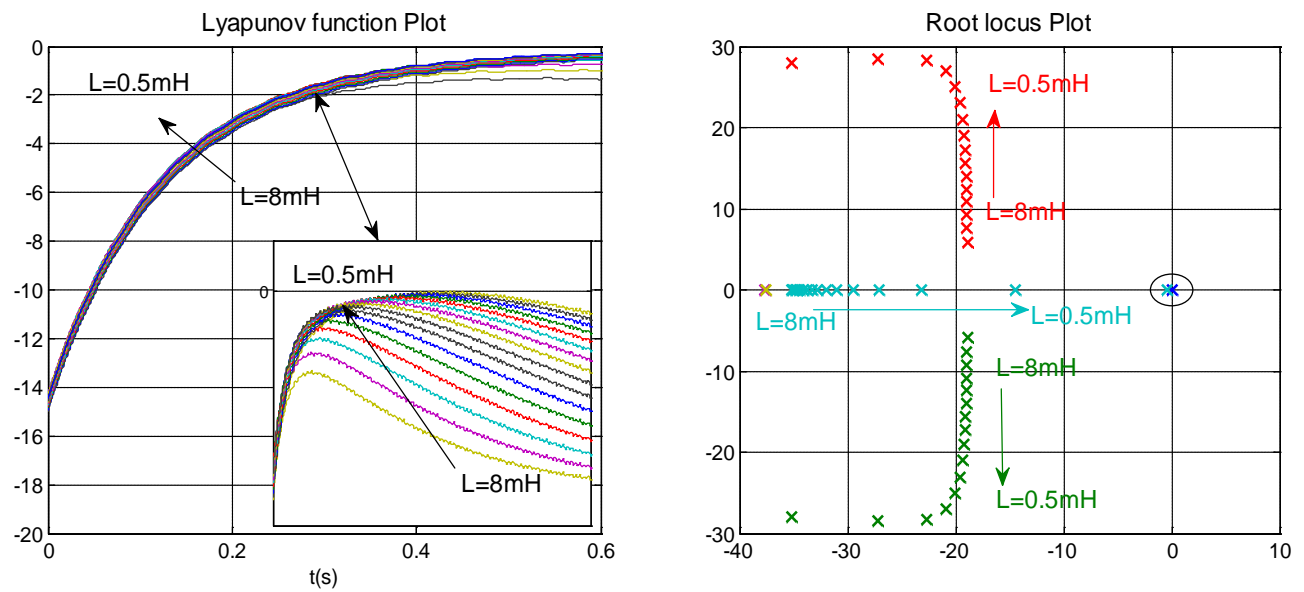

Fig. 5. Study of stability by means of Lyapunov function and root locus plot. 
Fig 5 s hows the study of stability by using the Lyapunov function and the root locus plot. They can be seen how the stability is affected when the line inductance decrease. The transient stability analysis by a Lyapunov method evaluate all variations starting in around a stable point that converge to it, therefore it can determine the lowest value of $V(X)$ on the surface $\dot{V}(X)=0$ and by this way the region of asymptotic stability is determined. The small signal stability analysis by a root locus plot shows how the roots are moved when the line inductor decreases, but cannot give an "a priori" region of asymptotic stability.

\section{Conclusions}

In this paper, a nonlinear state-space model of a Microgrid is presented. The model includes the most important dynamics. This modeling method is able to be extended to n-generators. The model has been analyzed by means of both stability studies Lyapunov function and root locus plot. A general methodology to find a valid Lyapunov function for non linear stability analysis has been presented. Using that Lyapunov function the region of asymptotic stability can be determined. These tools will allow the design of Microgrid systems with loads, generators and storage systems assuring the global stability of the system.

\section{References}

[1] A. G. Madureira, Coordinated voltage support in distribution networks with distributed generation and microgrids, IET Renewable Power Generation, 2009, pp. 439 - 454.

[2] P. N. Vovos, Centralized and Distributed Voltage Control: Impact on D istributed Generation Penetration, IEEE Transactions on Power Systems, 2007, pp. 476 - 483.

[3] C. L. Chen, State-space modeling, analysis, and implementation of paralleled inverters for microgrid applications, Applied Power Electronics Conference and Exposition (APEC), 2010, pp. $619-626$.

[4] A. Arulampalam, Control of power electronic interfaces in distributed generation microgrids, International Journal of Electronics, 2004, pp. $503-523$.

[5] N. Pogaku, Modeling, Analysis and Testing of Autonomous Operation of an InverterBased Microgrid, IEEE Transactions on Power Electronics, 2007, pp. 613 - 625.

[6] N. L. Soultanis, A Stability Algorithm for the Dynamic Analysis of Inverter Dominated Unbalanced LV Microgrids, IEEE Transactions on Power Systems, 2007, pp. 294 - 304.

[7] J. Guerrero, A wireless controller to enhance dynamic performance of parallel inverters in distributed generation system, IEEE Transactions on Power Electronics, 2004.

[8] P. Duminda, Stability Analysis of Microgrids with Constant Power Loads, Sustainable ICSET 2008. IEEE International Conference on Energy Technologies, 2008.

[9] E.A.A. Coelho, Small-Signal Stability for Parallel-Connected Inverters in Stand-Alone AC Supply Systems, IEEE Transactions on Industry Applications, 2002, pp. 533 - 542.

[10] A. Bacciotti, Liapunov Function and Stability in Control Theory, Springer, $2^{\text {nd }}$ edition, 2005 , pp. $27-80$.

[11] H. Khalil, Nonlinear Systems. Prentice Hall, $2^{\text {nd }}$ edition, 1996. 